\title{
Cracks in tension-field elastic sheets
}

\author{
O. Mahmood ${ }^{1}$. B. Audoly ${ }^{1,2}$, and S. Roux ${ }^{3 \dagger}$ \\ ${ }^{1}$ Sorbonne Universités, UPMC Univ Paris 06, CNRS, \\ Institut Jean Le Rond d'Alembert, 4 place Jussieu, 75005 Paris, France \\ ${ }^{2}$ LMS, École Polytechnique, CNRS, Univ. Paris-Saclay, 91128 Palaiseau, France \\ ${ }^{3}$ Laboratoire de Mécanique et Technologie, ENS Cachan / CNRS / Univ. Paris-Saclay, \\ 61 avenue du Président Wilson, 94235 Cachan Cedex, France
}

(Dated: September 7, 2018)

\begin{abstract}
We consider the deformation of a thin elastic sheet which is stiff in traction but very soft in compression, as happens in presence of wrinkling. We use the tension-field material model and explore numerically the response of a thin sheet containing multiple cracks of different geometries, when subjected to applied tension. With a single crack, the stress concentrates along a St-Andrew's cross-shaped pattern, whose branches extend from the crack tips to the corners of the domain; at a (small) distance $r$ from the crack tip, the stress displays the usual $r^{-1 / 2}$ stress singularity but with an unusual and non-universal angular dependence. A strong interaction between multiple cracks is reported and discussed: in particular, for certain configurations of the cracks, the tensile stiffness of a cracked sheet can be zero even though the sheet is made up of a single component.
\end{abstract}

The mechanics of plates, shells, foils or membranes is a fascinating topic exhibiting a variety of behaviors controlled by geometry rather than by the details of constitutive laws [1]. Thin elastic panels are prone to buckling under small compressive stress with a critical load going to zero in the membrane limit, i.e. as the thickness goes to zero. As a result, wrinkles are often encountered in membranes: load can be carried along 'tension rays' that are parallel to the directions of the wrinkles, while perpendicular direction remains soft [2]. Wrinkling introduces essential nonlinearity - hence major difficulties - in the modeling of thin membranes, both at the theoretical and numerical levels. As early as in 1938, Reissner [3] first formulated a 'tension-field theory' capturing tension rays in wrinkled regions. This pioneering work initiated a series of theoretical analyses on load transmission from rods, cables or point anchor to thin membranes [4 7]. In its simplest form, the tension field theory combines nonlinear geometry with a linear elastic response; Pipkin [8] showed that the tension field theory can be equivalently described by a non-linear constitutive law, derived from a relaxed strain energy: the constitutive law accounts for the wrinkles in an effective way, yielding different behaviors in traction and in compression. This mathematical approach was further extended to a 2D sheet embedded in a 3D space and to finite deformation [9. The tension field theory has been successfully used to predict the distribution of stress and wrinkles in very thin elastic plates [10, 11], and has served as a starting point to analyze the wavelength of wrinkles [12. Here, the mechanics of cracks in such a tension-field elastic sheet is investigated.

Unilateral materials can resist compression but not tension (e.g. granular media) or tension but not compression (e.g. fibrous materials). Unilaterality is one of the hallmarks of "non-smooth" mechanics [13]. It is associated with unusual properties, such as long-range interactions, which have been shown to be relevant to cell mechanosensing [14. In fact, the cancellation of an elastic stiffness depending on the state of strain breaks even the elliptic character of the governing equations. One of the most striking consequences of such a behavior is that entire regions are identically stress-free. Here, we show that cracks in a 'tension-field' medium display unusual features, including the presence of a diamond-shaped unloaded region surrounding the crack path, the occurrence of a cross pattern of wrinkles radiating from the crack, the channeling of stress into tension-rays, and strong interactions between cracks. The unilateral nonlinearity of the sheet renders the behavior of cracks structural, i.e. sensitive to the details of the domain geometry and of the boundary conditions.

We use a Pipkin-type of strain energy [8], which captures the loss in elastic stiffness in the direction perpendicular to wrinkles. Its derivation starts from the strain energy of classical linear elastic membranes $w_{l}\left(\varepsilon_{1}, \varepsilon_{2}\right)=$ $\frac{1}{2}(\lambda+2 \mu)\left(\varepsilon_{1}^{2}+\varepsilon_{2}^{2}\right)+\lambda \varepsilon_{1} \varepsilon_{2}$ in terms of the principal strains $\varepsilon_{i}$, corresponding to principal stress values $N_{1}^{l}=$ $\partial w_{l} / \partial \varepsilon_{1}=(\lambda+2 \mu)\left(\varepsilon_{1}+\nu \varepsilon_{2}\right)$ and $N_{2}^{l}=\partial w_{l} / \partial \varepsilon_{2}=$ $(\lambda+2 \mu)\left(\varepsilon_{2}+\nu \varepsilon_{1}\right)$, where $\nu=(1+2 \mu / \lambda)^{-1}$ is the $2 \mathrm{D}$ Poisson's ratio. The relaxed strain energy $w\left(\varepsilon_{1}, \varepsilon_{2}\right)$ is defined as $w_{l}\left(\varepsilon_{1}, \varepsilon_{2}\right)$ if $N_{1}^{l}>0$ and $N_{2}^{l}>0$ (taut case), as $w_{l}\left(\varepsilon_{1},-\nu \varepsilon_{1}\right)$ if $\varepsilon_{1}>0$ and $N_{2}^{l}<0$, as $w_{l}\left(-\nu \varepsilon_{2}, \varepsilon_{2}\right)$ if $N_{1}^{l}<0$ and $\varepsilon_{2}>0$, (wrinkled cases), and as 0 if $\varepsilon_{1}<0$ and $\varepsilon_{2}<0$ (slack case).

We introduce a convenient set of strain invariants, namely the strain magnitude $\varepsilon>0$ and the strain anisotropy $\phi(0 \leq \phi \leq \pi)$, defined by $\varepsilon_{m}=\varepsilon \cos \phi$ and $\varepsilon_{d}=\varepsilon \sin \phi$, where $\varepsilon_{m}=\operatorname{tr} \underline{\underline{\varepsilon}} / 2$ and $\varepsilon_{d}=(\operatorname{tr}[(\underline{\underline{\varepsilon}}-$ $\left.\left.\left.\varepsilon_{m} \underline{\underline{I}}\right)^{2}\right] / 2\right)^{1 / 2}$ are the mean and deviatoric strain. We focus on materials, such that $\lambda=\mu$ (i.e., $\nu=1 / 3$ ), that are attainable as the continuum limit of a discrete isotropic medium with linear elastic central force interactions. The 


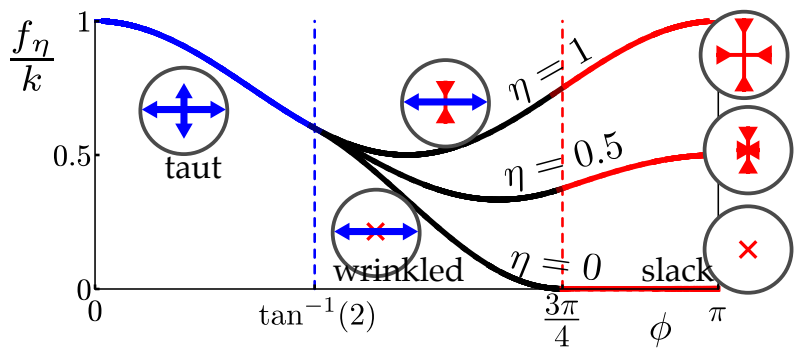

FIG. 1. Function $f_{\eta}(\phi)$ showing the dependence of the elastic energy density on the strain anisotropy $\phi$, for different values of the regularizing parameter $\eta$. For mild strain anisotropy $0<\phi<\tan ^{-1}(2)$ and tensile strains, the membrane is taut and all curves coincide. For large strain anisotropy, $\tan ^{-1}(2)<\phi<3 \pi / 4$, the membrane is wrinkled: one of the principal stress is compressive but goes to zero as $\eta \rightarrow 0$. For small strain anisotropy, $3 \pi / 4<\phi<\pi$, and contractile strains, both principal stress values are compressive, and vanish in the limit $\eta \rightarrow 0$. Arrows in circular insets denote the principal stress values; a red cross denote a zero principal stress, as obtained by relaxation in the limit $\eta \rightarrow 0$.

relaxed membrane energy density reads

$$
w(\epsilon, \phi)=4 \lambda \epsilon^{2} \times \begin{cases}\frac{3+\cos 2 \phi}{4} & \text { if } 0<\phi<\tan ^{-1}(2) \\ \frac{1+\sin 2 \phi}{3} & \text { if } \tan ^{-1}(2)<\phi<\frac{3 \pi}{4} \\ 0 & \text { if } \frac{3 \pi}{4}<\phi<\pi .\end{cases}
$$

This $w$ is not strictly convex and we regularize it by adding a fraction $\eta$ of the non-relaxed energy, $w_{\eta}(\epsilon, \phi)=$ $(1-\eta) w(\epsilon, \phi)+\eta w_{l}(\epsilon, \phi)$. The regularizing parameter $\eta$ varies in the range $0<\eta<1$, with $\eta=1$ corresponding to a classical linearly elastic (Hookean) medium, and the limit $\eta \rightarrow 0$ to a tension-field material. This regularized energy can be written as

$$
w_{\eta}(\varepsilon, \phi)=\frac{1}{2} \varepsilon^{2} f_{\eta}(\phi)
$$

where $f_{\eta}$ is a piecewise $\mathcal{C}^{2}$-smooth function of the strain anisotropy $\phi$, which is plotted in Fig. 1.

By differentiating $w_{\eta}$ with respect to the strain $\underline{\underline{\varepsilon}}$, one can derive the constitutive law. It is non-linear as soon as $\eta<1$ because of the essentially non-linear dependence on the strain anisotropy $\phi$ through the function $f_{\eta}$. However, the elastic energy $w_{\eta}$ is positively homogeneous of degree 2 with respect to the strain magnitude $\varepsilon$, see (1), and as a result the the stress $\underline{\underline{\sigma}}$ is positively homogeneous of degree 1 with respect to $\varepsilon$, as in linear elasticity.

In the following, a numerical modeling of such an elastic medium is proposed. The minimization of the integrated strain energy $\iint w_{\eta} \mathrm{d} x \mathrm{~d} y$ is implemented using the finite element library libmesh 15 . The tangent stiffness matrix is obtained from Eq. (1) by automatic differentiation [16]. Prescribed displacements are enforced by a penalty method. Equilibria are calculated in different geometries and for different values of the stiffness

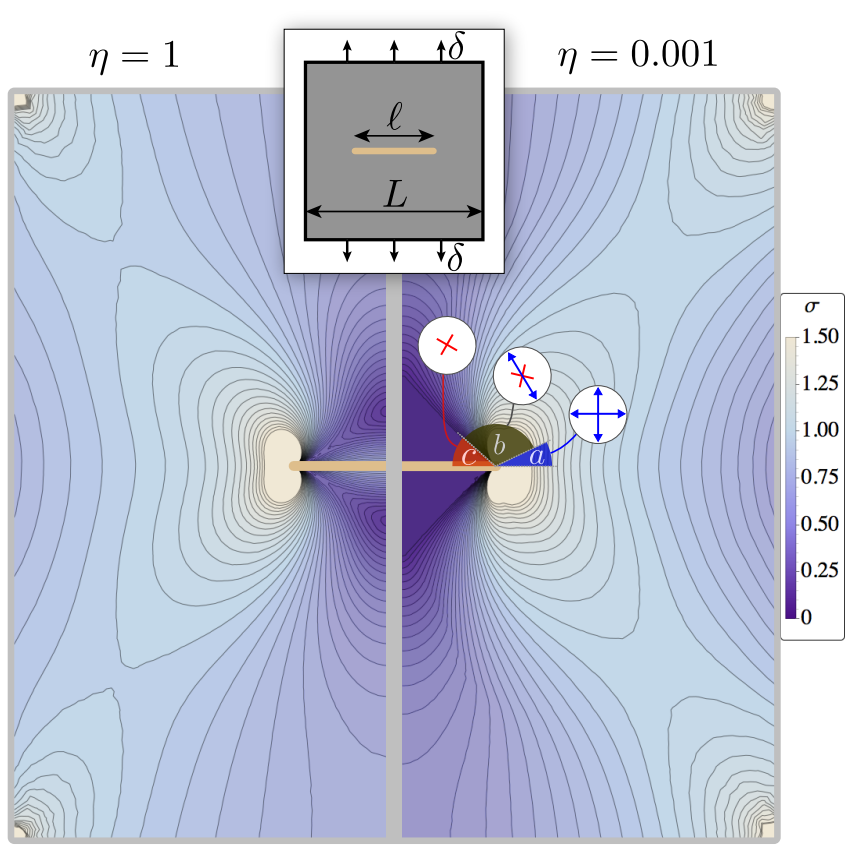

FIG. 2. Distribution of the stress intensity $\sigma=\|\underline{\sigma}\|$ in a square domain of size $L$ comprising a single centered crack (light brown line) with length $\ell=L / 4$. The stress distribution is symmetric and only one half of the square domain is shown: linearly elastic case $(\eta=1$, left $)$ and tension-field case $(\eta=$ $10^{-3}$, right). In the latter case, the principal stress values and directions are sketched near crack tip differ in the 3 angular sectors shown as overlays: (a) taut region, (b) wrinkled region, and (c) slack region. Away from crack tip, the sector (c) gives rise to a diamond-shaped region enclosing the crack path, where the stress drops to zero (dark purple central region, $\left.\eta=10^{-3}\right)$.

parameter $\eta, 0<\eta \leq 1$. Special attention is given to the behavior of the numerical solution in the singular limit $\eta \rightarrow 0$ where it convergences to a tension-field medium (this convergence is established numerically in Section 1 of the Supplementary Information).

The case of a square domain of size $L$ comprising a preexisting centered crack of size $\ell<L$, see Fig. 2, is first investigated. Prescribed displacements $\underline{u}=(0, \delta)$ and $\underline{u}=(0,-\delta)$ are imposed at the top and bottom boundaries, respectively; the lateral boundaries are tractionfree. Because of the symmetry, the crack is loaded in pure mode I. Comparison of the stress distribution in the linearly elastic case $(\eta=1$, left $)$ and close to the tension-field limit $\left(\eta=10^{-3}\right.$, right) reveals a distinctive feature in the limit $\eta \rightarrow 0$ : a diamond-like region surrounding the crack tip appears when $\eta \rightarrow 0$, where the membrane is slack (dark central diamond).

As there is no microscopic lengthscale in the problem, the stress must have a power-law singular behavior as a function of the distance $r$ to the crack tip, for any value of $\eta$. The numerical analysis of the stress singularity in Fig. 3 a confirms this, and shows furthermore that the 

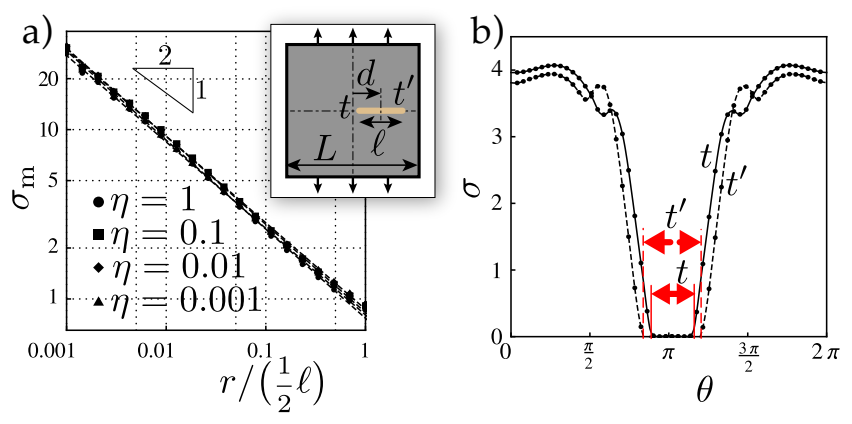

FIG. 3. Analysis of stress singularity near the tips of an interior crack with length $\ell=L / 4$. (a) Dependence of mean stress $\sigma_{m}=\operatorname{tr} \underline{\sigma} / 2$ on the distance to crack tip $r$ ahead of crack in the centered case $(d=0)$, for different values of the parameter $\eta$ : the power-law singularity $\sigma \sim r^{-1 / 2}$ is recovered for all values of $\eta$ but the stress intensity factor depends (mildly) on $\eta$. (b) Dependence of the stress intensity $\sigma$ on the polar variable $\theta$ along two small circles with radius $\ell / 40$ centered on the tips $t$ and $t^{\prime}$ of an off-center crack $(d=\ell / 2)$, with $\eta=10^{-3}$. The direction ahead of either crack tip corresponds to $\theta=0$. Even though both crack tips are loaded in pure mode I, the two plots of the stress are non-proportional; this illustrates the non-universality of the angular dependence of the stress near crack tips in mode I when $\eta<1$. The thick red arrows correspond to the slack region surrounding the crack, labelled 'c' in Fig. 2 .

scaling law $\sigma \sim r^{-1 / 2}$ from linear elasticity $(\eta=1)$ remains applicable to any value of $\eta$. Indeed, the path independence of the $J$-integral [17] holds for for non-linear elasticity problems such as this one, and implies a strain energy concentration as $w \sim 1 / r$; in Eq. 11, $w_{\eta} \sim \varepsilon^{2}$ and so $\varepsilon \sim \sigma \sim r^{-1 / 2}$ for any value of $\eta$. On the other hand, the angular dependence of the near-tip stress is not universal (and cannot be predicted by the linear membrane theory), as shown in Fig. 3b. In particular, the slack region $c$ adjacent to the crack tip in Fig. 2p, corresponding to the plateau $\sigma=0$ in Fig. $3 \mathrm{~b}$, has a different angular span in two crack tips from the same sample $\left(t \neq t^{\prime}\right)$.

The interaction between cracks is now analyzed in the geometry shown in Fig. 4a ; it comprises two cracks of equal length extending to the lateral boundaries. A uniform vertical displacement $\delta$ is again applied on the top and bottom boundaries. The equilibrium is solved numerically and the traction force $F_{T}$ is calculated. The equivalent stiffness $F_{T} / \delta$ is then plotted as a function of the regularizing parameter $\eta$. The log-log plot in Fig. 4a shows two radically different behaviors in the tensionfield limit $\eta \rightarrow 0$, depending on the length of the cracks. For cracks shorter than a critical length, $\ell<\ell_{\mathrm{c}}$, tensionrays connect the top and bottom boundaries (where the loading is applied) while avoiding the crack lips and the associated stress-free condition, see Fig. 4b: as a result, the equivalent stiffness converges to a non-zero value for $\eta \rightarrow 0$, see Fig. 4k. For cracks slightly longer than the critical length, $\ell>\ell_{\mathrm{c}}$, the behavior is markedly different:
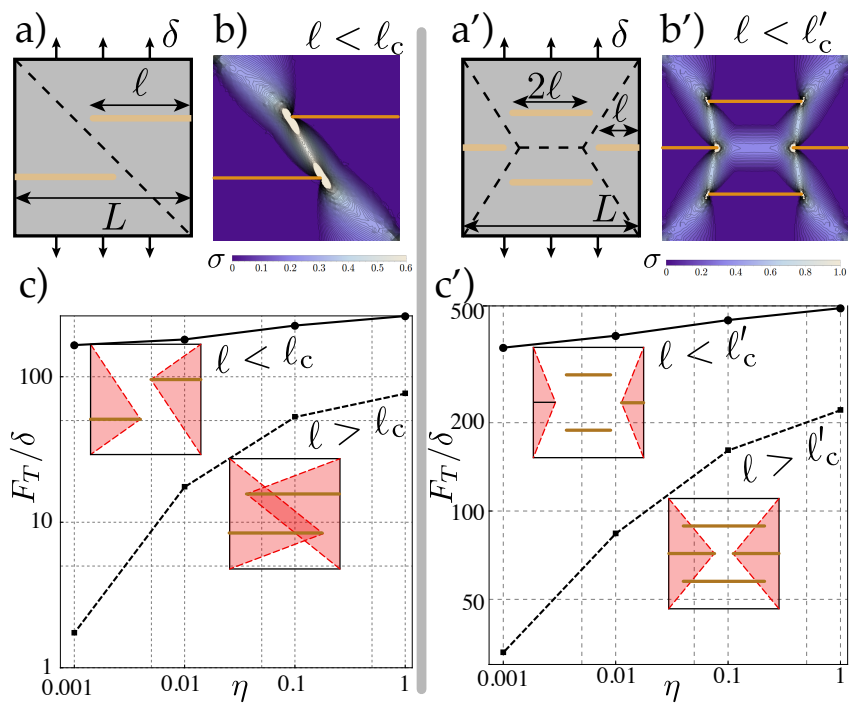

FIG. 4. Equivalent stiffness of samples comprising multiple cracks. (a,a'): geometry with 2 and 4 cracks having variable length. (b,b'): stress map for crack shorter than the critical length, $\ell<\ell_{\mathrm{c}}$ and $\ell<\ell_{\mathrm{c}}^{\prime}$, showing that the applied traction is mediated by tension-rays extending from the upper to the lower boundary in the tension-field limit, here with $\eta=0.01$. (c,c'): equivalent stiffness $F_{T} / \delta$ as a function of crack length $\ell$, where $F_{T}$ is the applied traction force and $\delta$ the imposed displacement: the stiffness is zero in the tension-field limit $\eta \rightarrow 0$ if the cracks are longer than a critical length set purely by geometry. Inset: convex hulls of simple components of the stress-free boundaries, where the membrane becomes slack in the limit.

the equivalent stiffness drops to zero in the tension-field limit $\eta \rightarrow 0$, see Fig. 怩: the crack sheet as a whole is no longer capable of sustaining traction. This sudden change in the response reveals a strong interaction between the cracks. The simulation shows that the ultimate tension-rays, for $\ell=\ell_{\mathrm{c}}$, is the diagonal of the square domain (dashes in Fig. 4 a): consistently, the critical crack length $\ell_{\mathrm{c}}$ is set by the purely geometric condition that the cracks make incipient contact with the diagonal.

The geometry comprising four cracks shown in Fig. 4 4 , shows both similarities and some subtle differences. For shorter cracks, $\ell<\ell_{\mathrm{c}}^{\prime}$, tension-rays connect the four corners (see Fig. $4 \mathrm{~b}^{\prime}$ ) and the equivalent stiffness remains finite in the tension-field limit $\eta \rightarrow 0$. For longer cracks, $\ell<\ell_{\mathrm{c}}^{\prime}$, it drops to zero. The critical length $\ell_{\mathrm{c}}^{\prime}$, however, does not correspond to the condition that the cracks meet with the diagonal of the domain. In fact, the ultimate tension-rays do not take place along the diagonal; instead, they form a network having five branches with a two-legs/body/two-arms topology. The critical length $\ell_{c}^{\prime}$ is set by the geometrical condition that such a network can be traced out in the domain without crossing the cracks.

The topology of the ultimate crack tension-ray graph - and, hence, the critical crack length - can be pre- 
dicted by a simple unifying geometrical rule. For the sample to be able to sustain a load in the tension-field limit, it must be possible to draw a path joining the top and bottom parts of the boundary (where a load is applied to enforce the prescribed displacement) without crossing any crack path nor entering in any of the convex hulls of the simply-connected components of the stress-free boundaries. Such convex hulls are shown in light red in the insets of Fig. 4,,c'; by adapting an argument for traction-loose (dry masonry) materials, one can indeed show that these convex hulls are free of stress in the tension-field limit [18. In the 2-cracks geometry in Fig. 4 a, the two convex hulls start to overlap at $\ell_{\mathrm{c}}$, and the ultimate stress channel passes in the narrow gap between them (which happens to lie on the diagonal). In the 4-cracks geometry in Fig. 4a', the two convex hulls make incipient contact with the interior cracks at $\ell_{c}^{\prime}$, and the five-branch topology of the tension-ray graph passes in the narrow gap between them as well, see insets in Fig. 4 f,c'. In earlier work on oscillatory cracks in torn membranes, convex hulls have been shown to play a fundamental role as well [19].

In the spirit of the early work of Pipkin [8, we have described the wrinkling of a membrane comprising cracks, when subjected to a macroscopic tensile-type of loading. To do so, we have used an effective non-linear constitutive law deriving from a relaxed strain energy. The stress concentration at crack tips has been first investigated. The usual power-law singularity predicted by the linear elastic fracture mechanics has been recovered, but the angular stress distribution, say, near a crack tip loaded in mode I, is no longer universal (by contrast with that of the classical linear fracture mechanics which, in pure mode I, is universal up to a stress intensity factor $K_{I}$ ). The universality is broken by the fact that the macroscopic loading and boundary conditions can affect the small-scale features of the solution through long-range interactions. Such interactions were also revealed by the analysis of a rectangular domain comprising several cracks: the global stiffness drops to zero as soon as networks of tension-rays avoiding cracks cease to exist, and this happens well before the sheet is fully cracked. A simple geometrical criterion for the vanishing of the stiffness has been proposed, depending on the layout and lengths of the cracks. All these results have been confirmed using a finite-element implementation of the relaxed strain energy. Overall, this emphasizes the fundamental role played by geometry in unilateral media.
O.M. is supported by a CIFRE grant from ANRT and Saint-Gobain Recherche. We thank R. Gy and X. Brajer for stimulating discussions.

* O.M.: mahmood@lmm.jussieu.fr

† S.R.: stephane.roux@ens-paris-saclay.fr

[1] B. Audoly and Y. Pomeau, Elasticity and geometry: from hair curls to the nonlinear response of shells (Oxford University Press, 2010).

[2] H. Wagner, Zeitschrift für Flugtechnik und Motorluftschiffahrt, 200 (1929).

[3] E. Reissner, in Proceedings of the Fifth International Congress on Applied Mechanics (1938) pp. 88-92.

[4] K. Kondo, T. Iai, S. Moriguti, and T. Murasaki, in Memoirs of the unifying study of the basic problems in engineering sciences by means of geometry, Vol. 1, edited by K. Kondo (Gakujutsu Bunken Fukyu-Kai, Tokyo, 1955) p. 417.

[5] M. Stein and J. M. Hedgepeth, Analysis of partly wrinkled membranes, Technical note D-813 (NASA, 1961).

[6] G. P. Cherepanov, Journal of Applied Mathematics and Mechanics 27, 405 (1963).

[7] E. H. Mansfield, Proceedings of the Royal Society of London A: Mathematical, Physical and Engineering Sciences 316, 269 (1970).

[8] A. C. Pipkin, IMA Journal of Applied Mathematics 36, 85 (1986).

[9] D. J. Steigmann, Proceedings of the Royal Society of London A: Mathematical, Physical and Engineering Sciences 429, 141 (1990).

[10] R. D. Schroll, M. Adda-Bedia, E. Cerda, J. Huang, N. Menon, D. Russell, K. B. Toga, D. Vella, and B. Davidovitch, Physical Review Letters 111, 014301 (2013).

[11] D. Vella, J. Huang, N. Menon, D. Russell, and B. Davidovitch, Physical Review Letters 114, 014301 (2015).

[12] B. Davidovitch, R. D. Schroll, D. Vella, M. Adda-Bedia, and E. Cerda, PNAS 108, 18227 (2011).

[13] J.-J. Moreau, in Non-smooth Mechanics and Applications, CISM courses and lectures, Vol. 302 (Springer, Wien, 1988).

[14] P. Rosakis, J. Nobohm, and G. Ravichandran, Journal of the Mechanics and Physics of Solids 85, 16 (2015).

[15] B. S. Kirk, J. W. Peterson, R. H. Stogner, and G. F. Carey, Engineering with Computers 22, 237 (2006).

[16] C. Bendtsen and O. Stauning, FADBAD, a flexible $\mathrm{C}++$ package for automatic differentiation, Tech. Rep. IMMREP-1996-17 (Technical University of Denmark, 1996).

[17] J. R. Rice, Journal of Applied Mechanics 35, 379 (1968).

[18] G. Del Piero, Meccanica 24, 150 (1989).

[19] B. Audoly, P. M. Reis, and B. Roman, Physical Review Letters 95, 025502 (2005). 